This item was submitted to Loughborough's Research Repository by the author.

Items in Figshare are protected by copyright, with all rights reserved, unless otherwise indicated.

\title{
Comparison between Kalman filter and Robust filter for vehicle handling dynamics state estimation
}

PLEASE CITE THE PUBLISHED VERSION

http://www.elecpubs.sae.org/NXT/gateway.dll?f=templates\&fn=default.htm

PUBLISHER

(C) SAE International

VERSION

VoR (Version of Record)

LICENCE

CC BY-NC-ND 4.0

\section{REPOSITORY RECORD}

Satria, Medy, and Matt C. Best. 2011. "Comparison Between Kalman Filter and Robust Filter for Vehicle Handling Dynamics State Estimation”. figshare. https://hdl.handle.net/2134/8396. 
This item was submitted to Loughborough's Institutional Repository (https://dspace.lboro.ac.uk/) by the author and is made available under the following Creative Commons Licence conditions.

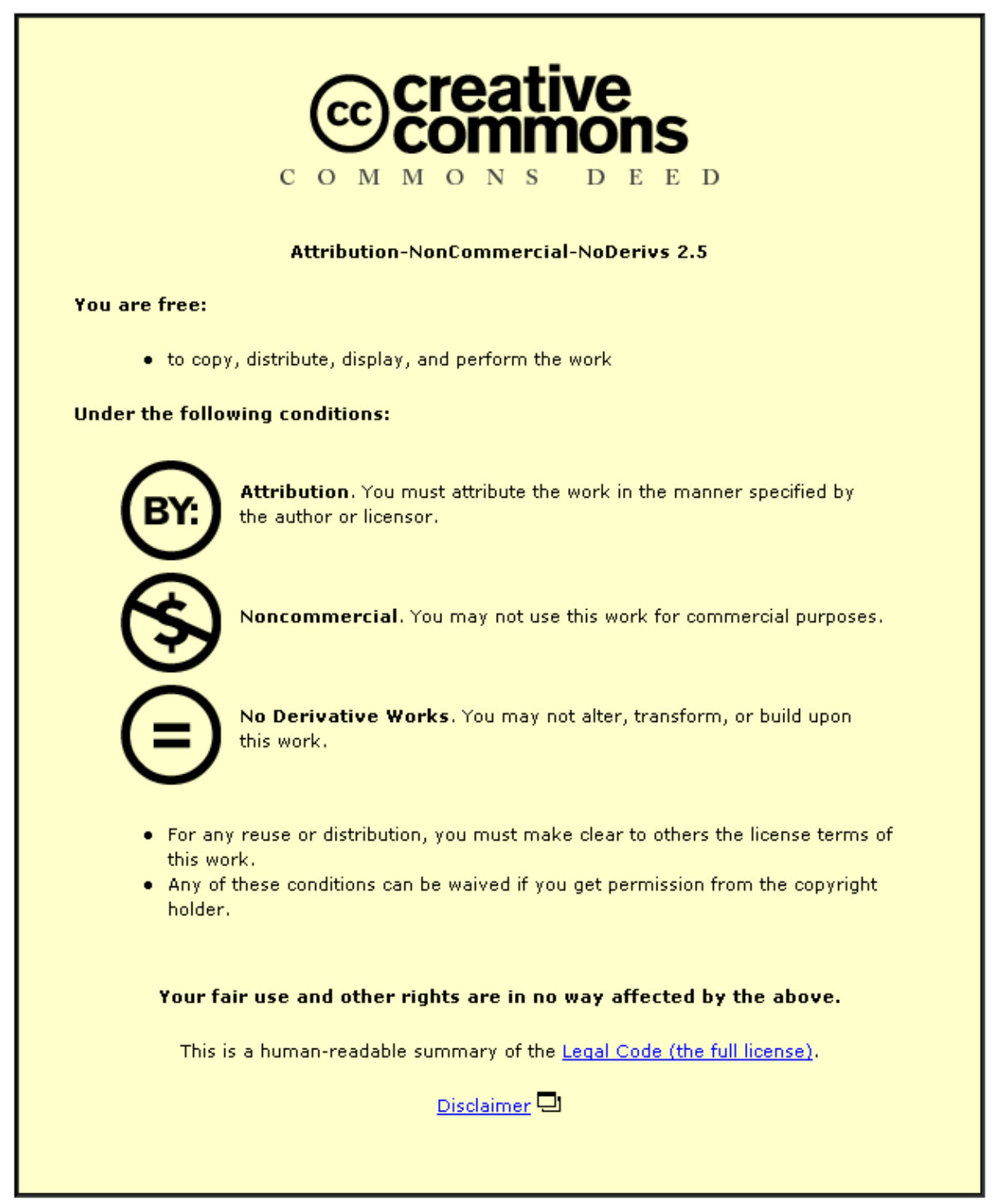

For the full text of this licence, please go to: http://creativecommons.org/licenses/by-nc-nd/2.5/ 


\section{Comparison Between Kalman Filter and Robust Filter for Vehicle Handling Dynamics State Estimation}

Medy Satria and Matthew C. Best

Loughborough University 
The appearance of this ISSN code at the bottom of this page indicates SAE'S consent that copies of the paper may be made for personal or internal use of specific clients. This consent is given on the condition, however, that the copier pay a per article copy fee through the Copyright Clearance Center, Inc. Operations Center, 222 Rosewood Drive, Danvers, MA 01923 for copying beyond that permitted by Sections 107 or 108 of the U.S. Copyright Law. This consent does not extend to other kinds of copying such as copying for general distribution, for advertising or promotional purposes, for creating new collective works, or for resale.

Quantity reprint rates can be obtained from the Customer Sales and Satisfaction Department.

To request permission to reprint a technical paper or permission to use copyrighted SAE publications in other works, contact the SAE Publications Group.

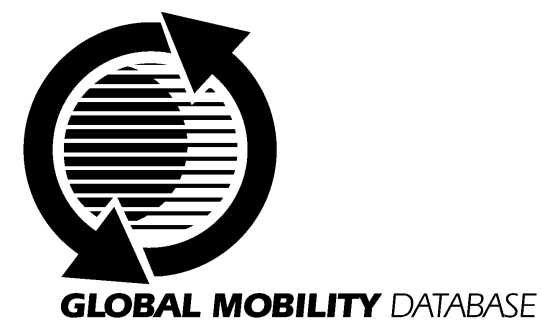

All SAE papers, standards, and selected books are abstracted and indexed in the Global Mobility Database

No part of this publication may be reproduced in any form, in an electronic retrieval system or otherwise, without the prior written permission of the publisher.

\section{ISSN 0148-7191}

Copyright $\odot 2002$ Society of Automotive Engineers, Inc.

Positions and opinions advanced in this paper are those of the author(s) and not necessarily those of SAE. The author is solely responsible for the content of the paper. A process is available by which discussions will be printed with the paper if it is published in SAE Transactions. For permission to publish this paper in full or in part, contact the SAE Publications Group.

Persons wishing to submit papers to be considered for presentation or publication through SAE should send the manuscript or a 300 word abstract of a proposed manuscript to: Secretary, Engineering Meetings Board, SAE.

\section{Printed in USA}




\title{
Comparison between Kalman Filter and Robust Filter for Vehicle Handling Dynamics State Estimation
}

\author{
Medy Satria and Matthew C Best \\ Loughborough University
}

Copyright (C) 2002 Society of Automotive Engineers, Inc.

\section{ABSTRACT}

This paper explores design methods for a vehicle handling dynamics state estimator based on a linear vehicle model. The state estimator is needed because there are some states of the vehicle that cannot be measured directly, such as sideslip velocity, and also some which are relatively expensive to measure, such as roll and yaw rates. Information about the vehicle states is essential for vehicle handling stability control and is also valuable in chassis design evaluation.

The aim of this study is to compare the performance of a Kalman filter with that of a robust filter, under conditions which would be realistic and viable for a production vehicle. Both filters are thus designed and tested with reference to a higher order source model which incorporates nonlinear saturating tyre force characteristics. Also, both filters rely solely on accelerometer sensors, which are simulated with expected noise characteristics in terms of amplitude and spectra.

As is widely known, the Kalman filter is a stochastic filter whose design depends on the nominal vehicle model and statistical information of process and measurement noises. By contrast, the robust filter is deterministic, formulated in terms of model parameter uncertainties and the expected gain of process and measurement noises. The objective of both filter designs is to minimise the variance of the estimation error. Both filters are designed to compensate the vehicle model non-linearities, parameter uncertainties and other modeling errors, which are represented in terms of process and measurement noise covariances in Kalman filter design and in terms of additive model uncertainties in robust filter design.

The study shows that the robust filter offers higher performance potential. The work concludes with a discussion on the practical realisation of each method, and gives recommendations for further research into a single design methodology which combines the benefits of both approaches.

Keywords: Vehicle Handling Dynamics, Kalman Filter, Robust Filter, State Estimator

\section{INTRODUCTION}

Recent research in advanced control techniques has given rise to the development of controllers which improve vehicle lateral handling stability [1], longitudinal handling stability [2], ride comfort [3], and also overall vehicle performance via integrated vehicle control [1, 4]. Many of these are model based multivariable control methods. Such controller designs need accurate information about vehicle states, such as sideslip velocity, yaw rate, roll rate, and roll angle. However, not all of the vehicle states can be measured directly (such as sideslip velocity) and also some of them are relatively expensive to measure directly, such as yaw and roll rates. Designing a model-based observer might best solve these problems.

A number of studies have been done in state observer design with many methods and many objectives, but still relatively few studies have been done to estimate a broad range of vehicle states. For example in [5], studies only focused on slip angle estimation for direct yaw moment control purpose. A wider state vector estimation was proposed by Kiencke and Daiß [6] using linear and non-linear Luenberger filters to estimate vehicle lateral states during cornering. However this did not capture the roll state of the vehicle and the method did not compensate the vehicle model uncertainties. Similar with [6], Venhovens and Naab [7] proposed a Kalman filter for lateral state estimation for BMW Driver Assistance Systems, but this paper has similar shortcomings.

This study is based on previous research that has been done by Best and Gordon [8,9]. Best and Gordon have proposed an extended adaptive Kalman filter to estimate the sideslip/roll/yaw states of the vehicle using two sets of accelerometer sensor. The result is accurate in a wide range of operating conditions because it utilises the non-linear model of the vehicle directly, however this method needs high computational ability, which may cause difficulty in real-time implementation. This study considers a less complicated algorithm using linear filters while attempting to capture a wide range of operating condition within certain robustness bound. The basic requirement is that the filter takes into account vehicle model uncertainties in its design process. A Kalman filter and a robust filter are 
examined because both of these filters have the ability to take into account vehicle model uncertainties although using different assumption about their source. Both the Kalman and robust filters are designed within the same operating condition in order to give comparable results.

\section{VEHICLE MODELS}

NON-LINEAR SOURCE MODEL - The simulation is carried out using a reference nonlinear vehicle model to provide 'true' state trajectories, sensor measurements and also data for parameter identification. This is formulated using four degrees of freedom comprising two translational motions (longitudinal and lateral) and two rotational motions (roll and yaw), described using vehicle centred SAE axes with roll motion is assumed to be constrained about an inclined roll axis (Figure 1). The vehicle is assumed to be moving on a flat road and operated under relatively constant forward velocity such that the vertical and pitch motions are supposed to be negligible. It operates using front wheel drive (FWD) and front wheel steer (FWS) system. The complete model can be described with the Newton-Euler equations as follow:

Longitudinal dynamics:

$$
\sum_{i=1}^{2} F_{x i}=M(\dot{u}-r v-r p h \cos \varepsilon)
$$

Lateral dynamics:

$$
\sum_{i=1}^{4} F_{y i}=M(\dot{v}+\dot{p} h \cos \varepsilon+r u)
$$

Roll dynamics:

$\left(\bar{I}_{x x} \cos \varepsilon-I_{x z} \sin \varepsilon\right) \dot{p}-I_{x z} \dot{r}+M h(\dot{v}+r u)=-\left(K_{f} \frac{s_{f}^{2}}{2}+K_{r} \frac{s_{r}^{2}}{2}+K_{s t a b}\right) \theta$

$-\left(B_{f} \frac{s_{f}^{2}}{2}+B_{r} \frac{s_{r}^{2}}{2}\right) p+\left(h_{r f}-h_{o}\right) \sum_{i=1}^{2} F_{y i}+\left(h_{r r}-h_{o}\right) \sum_{i=3}^{4} F_{y i}$

$\dot{\theta}=p$

Yaw dynamics:

$\left(I_{z z} \sin \varepsilon-I_{x z} \cos \varepsilon\right) \dot{p}+I_{z z} \dot{r}=b \sum_{i=1}^{2} F_{y i}-c \sum_{i=3}^{4} F_{y i}+\left(F_{x 1}-F_{x 2}\right) \frac{t_{f}}{2}$

The tyre forces generated in this system consist of 2 longitudinal tyre forces (at the front wheels) and 4 lateral tyre forces. Figure 2 shows the vehicle plan view and axis system that describes the detail of all longitudinal and lateral tyre forces generated in the vehicle under FWD and FWS system.

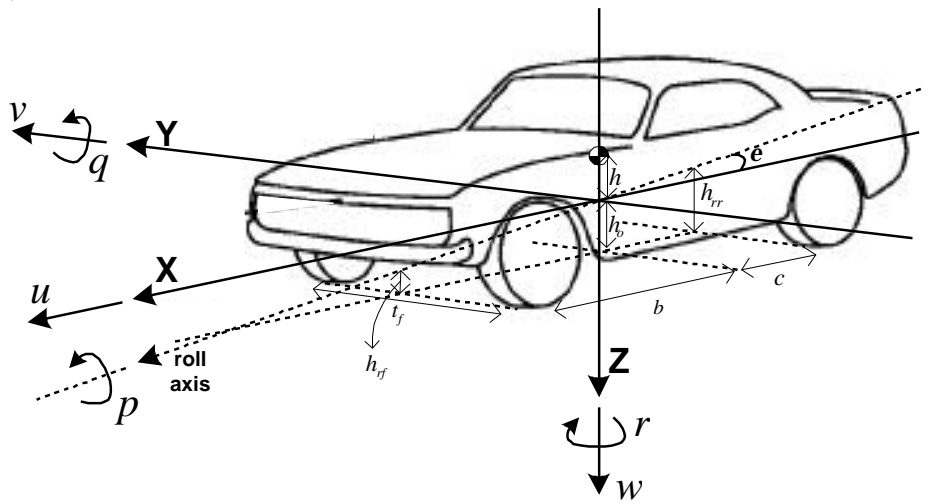

$$
\begin{array}{ll}
p: \text { Roll velocity } & u: \text { Longitudinal velocity (constant) } \\
q: \text { Pitch velocity (zero) } & v: \text { Sideslip velocity } \\
r: \text { Yaw velocity } & w: \text { Vertical velocity (zero) }
\end{array}
$$

Figure 1 : Vehicle axis system

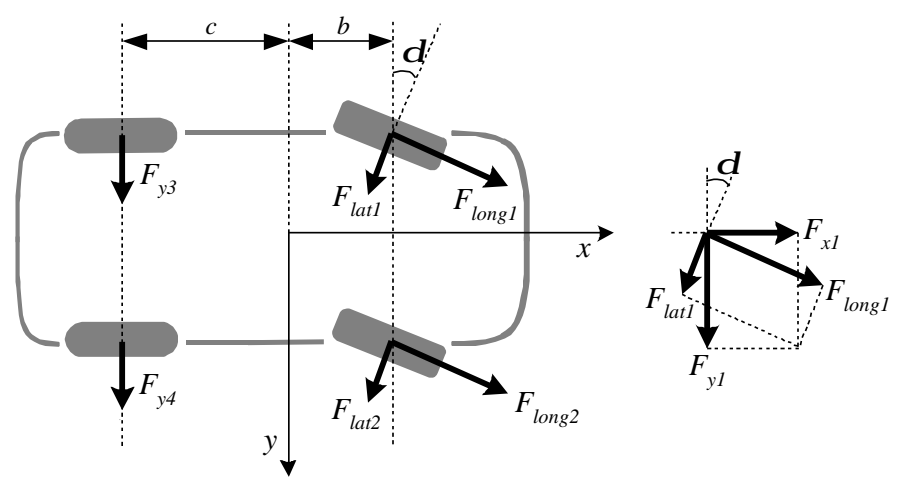

Figure 2 : Vehicle tyre forces

The longitudinal and lateral tyre forces at front wheels are formulated using combined slip characteristic Pacejka Tyre Magic Formula (as can be seen in [10]) as follows:

$$
F_{\text {long } 1,2}=\frac{\mu_{x} \cdot Z_{1,2} \cdot R_{1,2} \cdot S R_{1,2}}{\sqrt{S R_{1,2}^{2}+\mu_{1,2}^{2} \cdot \tan ^{2} \alpha_{1,2}}} F_{\text {lat }, 2}=\frac{\mu_{y} \cdot Z_{1,2} \cdot R_{1,2} \cdot \mu_{1,2} \cdot \tan \alpha_{1,2}}{\sqrt{S R_{1,2}^{2}+\mu_{1,2}^{2} \cdot \tan ^{2} \alpha_{1,2}}}
$$

where

$$
\begin{gathered}
R_{1,2}=D^{\prime} \cdot \sin \left(C^{\prime} \cdot \arctan \left(B^{\prime}\left(1-E^{\prime}\right) k_{1,2}+E^{\prime} \arctan \left(B^{\prime} \cdot k_{1,2}\right)\right)\right) \\
k_{1,2}=\sqrt{\bar{\alpha}_{1,2}^{2}+S R N_{1,2}^{2}} \quad \bar{\alpha}_{1,2}=\frac{C_{\alpha} \cdot \tan \left(\alpha_{1,2}\right)}{\mu_{y} \cdot Z_{1,2}} \\
S R N_{1,2}=\frac{K_{x} \cdot S R_{1,2}}{\mu_{x} \cdot Z_{1,2}} S R_{1,2}=\frac{w-u \cos \left(\alpha_{1,2}\right)}{u \cos \left(\alpha_{1,2}\right)} \\
\mu_{0}=\frac{C_{\alpha} \cdot \mu_{x}}{K_{x} \cdot \mu_{y}} \quad \mu_{1,2}=\left\{\begin{array}{l}
0.5\left(1+\mu_{0}\right)-0.5\left(1-\mu_{0}\right) \cos \left(\frac{k_{1,2}}{2}\right) \text { for }\left|\mathrm{k}_{1,2}\right| \leq 2 \pi \\
1 \text { for }\left|\mathrm{k}_{1,2}\right|>2 \pi
\end{array}\right.
\end{gathered}
$$

* Figure adapted from an original in 'Race Car Vehicle Dynamics', W. F. Milliken and D. L. Milliken 
and the sideslip angles at front wheels are:

$\alpha_{1}=\arctan \left(\frac{-(v+b r)}{\left(u+r \frac{t_{f}}{2}\right)}\right)+\delta \alpha_{2}=\arctan \left(\frac{-(v+b r)}{\left(u-r \frac{t_{f}}{2}\right)}\right)+\delta$

The steer angle results in longitudinal and lateral tyre forces as follows:

$$
\begin{aligned}
& F_{x 1,2}=F_{\text {long } 1,2} \cos \delta-F_{\text {lat } 1,2} \sin \delta \\
& F_{y 1,2}=F_{\text {long } 1,2} \sin \delta+F_{\text {lat } 1,2} \cos \delta
\end{aligned}
$$

Lateral tyre forces at the rear wheels are formulated using pure slip characteristic Pacejka Tyre Magic Formula as follows:

$$
F_{y 3,4}=\bar{F}_{y 3,4} \cdot \mu_{y} \cdot Z_{3,4}
$$

where

$$
\begin{gathered}
\bar{F}_{y 3,4}=D^{\prime} \cdot \sin \left(C^{\prime} \cdot \arctan \left(B^{\prime}\left(1-E^{\prime}\right) \cdot \bar{\alpha}_{3,4}+E^{\prime} \cdot \arctan \left(B^{\prime} \cdot \bar{\alpha}_{3,4}\right)\right)\right) \\
\bar{\alpha}_{3,4}=\frac{C a \cdot \tan \left(\alpha_{3,4}\right)}{\mu_{y} \cdot Z_{3,4}}
\end{gathered}
$$

and the sideslip angles at rear wheels are:

$\alpha_{3}=\arctan \left(\frac{-(v-c r)}{\left(u+r \frac{t_{r}}{2}\right)}\right) \alpha_{4}=\arctan \left(\frac{-(v-c r)}{\left(u-r \frac{t_{r}}{2}\right)}\right)$

The load transfer due to longitudinal and lateral tyre forces are formulated as follows:

Longitudinal load transfer:

$$
\begin{aligned}
& Z_{f}=\frac{c M g-\left(F_{x 1}+F_{x 2}\right) h_{o}}{b+c} \\
& Z_{r}=\frac{c M g+\left(F_{x 1}+F_{x 2}\right) h_{o}}{b+c}
\end{aligned}
$$

Lateral load transfer:

$$
\begin{aligned}
& Z_{1}=\frac{1}{t_{f}}\left[\left(F_{y 1}+F_{y 2}\right) h_{r f}-\left(K_{f} \frac{s_{f}^{2}}{2} \theta+K_{s t a b} \theta+B_{f} \frac{s_{f}^{2}}{2} p\right)+Z_{f} \frac{t_{f}}{2}\right] \\
& Z_{3}=\frac{1}{t_{r}}\left[\left(F_{y 3}+F_{y 4}\right) h_{r r}-\left(K_{r} \frac{s_{r}^{2}}{2} \theta+B_{r} \frac{s_{r}^{2}}{2} p\right)+Z_{r} \frac{t_{r}}{2}\right] \\
& Z_{2}=Z_{f}-Z_{1} Z_{4}=Z_{r}-Z_{3}
\end{aligned}
$$

In this case, both the steer angle $\delta$ and the driven wheel speed $w$ are assumed to be available as deterministically known inputs. The vehicle model is therefore completely described in terms of five state variables, 25 constant parameters and two inputs, summarised in Table 1.

The measurable variables are taken as four lateral accelerometers positioned as shown in Figure 3. The main reason for choosing accelerations other than to ensure observability is that they are cheap and readily available.

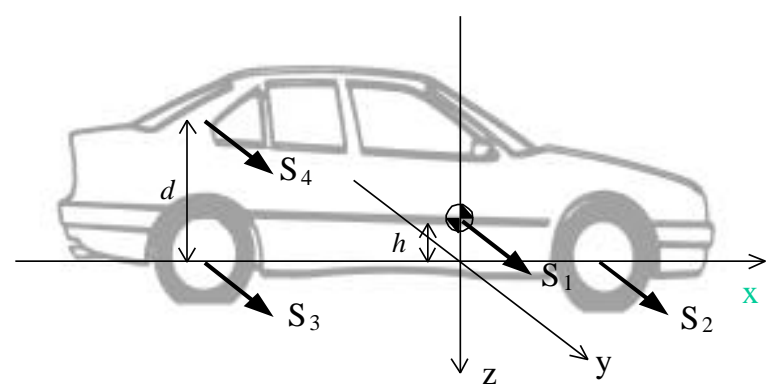

Figure 3 : Sensor placement and orientation

All the sensors are oriented laterally, and they are modeled as follows:

$$
\begin{gathered}
s_{1}=\dot{v}+h \dot{p} \cos \varepsilon+r u+\varepsilon_{1} \quad s_{2}=\dot{v}+b \dot{r}+r u+\varepsilon_{2} \\
s_{3}=\dot{v}-c \dot{r}+r u+\varepsilon_{3} \quad s_{4}=\dot{v}+d \dot{p} \cos \varepsilon-c \dot{r}+r u+\varepsilon_{4}
\end{gathered}
$$

It can be seen from equation (12) that a combination of some or all of the sensors can completely contain the state information such that the system will be completely observable. In each case, the sensor signal includes measurement noise which is simulated using an independent gaussian white noise signal with equal magnitude on each sensor:

$$
\varepsilon_{i}=N\left(0,[2 \rho]^{2}\right)
$$

with $\rho$ used as an amplification factor such that the noise levels are referenced to a nominal lateral acceleration of $2 \mathrm{~m} / \mathrm{s}^{2}$.

The non-linear vehicle model can be formulated in terms of non-linear state space equation using the explicit form of equation (1) to (4) for vehicle state dynamics and equation (12) for vehicle sensor dynamics. The non-linear state space equations are then:

$$
\begin{aligned}
\dot{\vec{x}}(t) & =f(\vec{x}(t), \vec{u}(t), t) \\
\vec{y}(t) & =g(\vec{x}(t), \vec{u}(t), t)
\end{aligned}
$$

where $\vec{x}=[u, v, p, r, \theta]^{T}$ is the vehicle state variable vector, $\vec{u}=[\delta, w]^{T}$ is the vehicle input variable vector and $\vec{y}=\left[s_{1}, \ldots, s_{4}\right]^{T}$ is the sensor measurement vector which depends on the sensor set chosen in the 
simulation. This non-linear model is the source vehicle model for the simulation.

\begin{tabular}{|c|c|}
\hline \multicolumn{2}{|c|}{ States, $\mathrm{x}$} \\
\hline $\mathrm{u}$ & forward velocity $(\mathrm{m} / \mathrm{s})$ \\
\hline $\mathrm{v}$ & sideslip velocity $(\mathrm{m} / \mathrm{s})$ \\
\hline $\mathrm{p}$ & roll angular velocity ( $\mathrm{rad} / \mathrm{s})$ \\
\hline $\mathrm{r}$ & yaw angular velocity ( $\mathrm{rad} / \mathrm{s}$ ) \\
\hline$\theta$ & roll angle ( $\mathrm{rad})$ \\
\hline \multicolumn{2}{|c|}{ Parameters, $\eta$ (default value) } \\
\hline $\mathrm{I}_{\mathrm{xx}}$ & roll moment of inertia $\left(600 \mathrm{kgm}^{2}\right)$ \\
\hline $\mathrm{I}_{\mathrm{zz}}$ & yaw moment of inertia $\left(1300 \mathrm{kgm}^{2}\right)$ \\
\hline$I_{x z}$ & roll/yaw cross moment of inertia $\left(80 \mathrm{kgm}^{2}\right)$ \\
\hline $\mathrm{m}$ & vehicle mass $(830 \mathrm{~kg})$ \\
\hline b & longitudinal distance of CG to front axle $(1.1 \mathrm{~m})$ \\
\hline c & longitudinal distance of CG to rear axle $(1.4 \mathrm{~m})$ \\
\hline h & CG height above roll axis $(0.468 \mathrm{~m})$ \\
\hline$h_{0}$ & ground plane to roll axis distance below CG $(0.332 \mathrm{~m})$ \\
\hline$h_{r f}$ & ground to roll axis vertical distance at front axle $(0.2 \mathrm{~m})$ \\
\hline$h_{\mathrm{rr}}$ & ground to roll axis vertical distance at rear axle $(0.5 \mathrm{~m})$ \\
\hline$\varepsilon$ & inclined angle between roll axis and $\mathrm{x}$-axis $(6.8428 \mathrm{deg}$ ) \\
\hline$t_{f}$ & front track $(1.5 \mathrm{~m})$ \\
\hline $\mathrm{t}_{\mathrm{r}}$ & rear track $(1.5 \mathrm{~m})$ \\
\hline$s_{f}$ & front suspension track $(1.2 \mathrm{~m})$ \\
\hline $\mathrm{s}_{\mathrm{r}}$ & rear suspension track $(1.2 \mathrm{~m})$ \\
\hline $\mathrm{K}_{\mathrm{f}}$ & front suspension stiffness $(20 \mathrm{kN} / \mathrm{m})$ \\
\hline $\mathrm{K}_{\mathrm{r}}$ & rear suspension stiffness $(22 \mathrm{kN} / \mathrm{m})$ \\
\hline $\mathrm{K}_{\text {stab }}$ & front stabiliser bar stiffness (22918 Nm/rad) \\
\hline$B_{f}$ & front suspension damping rate $(1100 \mathrm{Ns} / \mathrm{m})$ \\
\hline $\mathrm{B}_{\mathrm{r}}$ & rear suspension damping rate $(1200 \mathrm{Ns} / \mathrm{m})$ \\
\hline$S_{p}$ & $\begin{array}{l}\text { Pacejka tyre model shape coefficients }\left(B^{\prime}=0.714, C^{\prime}=1.4, D^{\prime}=1 \text {, }\right. \\
\left.E^{\prime}=-0.2\right)\end{array}$ \\
\hline $\mathrm{C}_{\alpha}$ & zero lateral slip cornering stiffness ( $35 \mathrm{kN} / \mathrm{rad}$ ) \\
\hline $\mathrm{K}_{\mathrm{x}}$ & zero longitudinal tyre slip rate $(35 \mathrm{kN})$ \\
\hline$\mu_{x}$ & Tyre longitudinal friction coefficient (1.0) \\
\hline$\mu_{y}$ & Tyre lateral friction coefficient $(1.0)$ \\
\hline \multicolumn{2}{|c|}{ Inputs, u } \\
\hline$\delta$ & front wheel steer angle $(\mathrm{rad})$ \\
\hline w & front wheel rotation velocity referred to tyre contact path $(\mathrm{m} / \mathrm{s})$ \\
\hline
\end{tabular}

Table 1 : Model nomenclature

LINEAR VEHICLE MODEL - The linear vehicle model is defined by linearisation of the non-linear vehicle model at its certain operating condition. In this simulation, the operating condition of the vehicle is set under constant forward speed and wheel speed. Under this operating condition, the order of the linear model is reduced to four states and one input. The linear model of the vehicle is then formulated as a linear time invariant state space equation as follows:

$$
\begin{aligned}
& \dot{\vec{x}}(t)=A \vec{x}(t)+B \vec{u}(t) \\
& \vec{y}(t)=C \vec{x}(t)+D \vec{u}(t)
\end{aligned}
$$

where $\vec{x}=[v, p, r, \theta]^{T}, \vec{u}=[\delta]$ and $\vec{y}=\left[s_{1}, \ldots, s_{4}\right]^{T}$. The parameters of the state space above $(A, B, C$ and $D$ matrices) can be obtained either using Jacobian method (analytic) or least square estimation (LSE) method (numeric). In this study, the LSE method is chosen because it will give more accurate result than Jacobian method due to the ability of the LSE method to capture a wider operating condition rather than Jacobian method.

Using the LSE method, the parameters of the linear model can be obtained from a simple linear regression of input-output source data taken from the source model. Given a set of N input data $\left(\Phi_{N}\right)$ and $N$ output data $\left(Y_{N}\right)$, the LSE method can be used by:

$$
\begin{gathered}
Y_{N}=\Phi_{N} \theta+e \\
\hat{\theta}=\left(\Phi_{N}^{T} \Phi_{N}\right)^{-1} \Phi_{N}^{T} Y_{N}
\end{gathered}
$$

where $\theta$ is the 'true' parameters and $\hat{\theta}$ is the estimated parameters. A and $\mathrm{B}$ matrices can be got by setting $\Phi_{N}=\left[\begin{array}{ll}\vec{x}^{T} & \vec{u}^{T}\end{array}\right], \quad Y_{N}=\dot{\vec{x}}^{T} \quad$ and $\quad \theta=\left[\begin{array}{ll}A & B\end{array}\right]^{T}$. Respectively, $C$ and $D$ matrices can be got by setting $\Phi_{N}=\left[\begin{array}{ll}\vec{x}^{T} & \vec{u}^{T}\end{array}\right], Y_{N}=\vec{y}^{T}$ and $\theta=\left[\begin{array}{ll}C & D\end{array}\right]^{T}$.

The source data for $\Phi_{N}$ and $Y_{N}$ are generated by simulation of the source model using the following steering input, chosen with a view to feasible vehicle tests, to induce a high level of state dynamic variation within the achievable frequency response envelope of a test driver:

$$
\delta(t)=N\left(0, \sigma_{\delta}^{2}\right)
$$

bandlimited $0-5 \mathrm{~Hz}, \sigma_{\delta}=0.0184 \mathrm{rad}(1.0558 \mathrm{deg})$

The data are collected for a 100-second data batch.

\section{KALMAN FILTER DESIGN}

The Kalman filter is a stochastic filter based on a linear plant model and a-priori information of process and measurement noises. The design engineer assumes that the plant to be observed is influenced by random process noise $\vec{w}(t)$ that is characterised by stochastic quantities and that the sensors used are corrupted by random measurement noise $\vec{v}(t)$ as well. The principle of estimating the system states $\vec{x}(t)$ is based on a comparison of measured outputs $\vec{y}(t)$ and estimated outputs $\vec{y}_{e}(t)$. The difference $\vec{e}_{y}=\vec{y}(t)-\vec{y}_{e}(t)$ is fed back to the filter through the Kalman innovation gain $K$ to update the estimated states $\vec{x}_{e}(t)$.

Consider a linear time invariant vehicle model influenced by process and measurement noises as follow:

$$
\begin{aligned}
& \dot{\vec{x}}(t)=A \vec{x}(t)+B \vec{u}(t)+\vec{w}(t) \\
& \vec{y}(t)=C \vec{x}(t)+D \vec{u}(t)+\vec{v}(t)
\end{aligned}
$$

where $\vec{w}(t)$ and $\vec{v}(t)$ are process and measurement noises which are assumed to be white noise, gaussian and to have zero mean, with stochastic information formulated as follow:

$$
\begin{array}{cc}
E\left[\vec{w}(t) \vec{w}^{\prime}(\tau)\right]=Q \delta(t-\tau) & E[\vec{w}(t)]=0 \\
E\left[\vec{v}(t) \vec{v}^{\prime}(\tau)\right]=R \delta(t-\tau) & E[\vec{v}(t)]=0 \\
E\left[\vec{w}(t) \vec{v}^{\prime}(\tau)\right]=S \delta(t-\tau)
\end{array}
$$


where $Q$ and $R$ are covariance matrices that must be symmetric and non negative definite and $S$ is cross covariance matrix representing correlations between $\vec{w}(t)$ and $\vec{v}(t)$. The objective of the filtering problem is to minimise the estimation error variance defined as:

$$
E\left\{\left[\vec{x}(t)-\vec{x}_{e}(t)\right]\left[\vec{x}(t)-\vec{x}_{e}(t)\right]^{\prime}\right\}=P(t)
$$

Then a Kalman filter can be designed with following structure:

$$
\begin{gathered}
\dot{\vec{x}}_{e}(t)=A \vec{x}_{e}(t)+B \vec{u}(t)+K_{k}\left(\vec{y}(t)-C \vec{x}_{e}(t)-D \vec{u}(t)\right) \\
K_{k}=\left(P C^{\prime}+S\right) R^{-1}
\end{gathered}
$$

where $K_{k}$ is the Kalman filter gain and $\mathrm{P}$ is the steady state solution of the algebraic Riccati equation as follow:

$$
A P+P A^{\prime}-\left(P C^{\prime}+S\right) R^{-1}\left(C P+S^{\prime}\right)+Q=0
$$

Problem arises in the use of Kalman filter because of the above assumptions about expected error signal. Modeling errors due to tyre force non-linearity pose a particular risk. Figure 4 summarises the modeling errors which arise in observer design for practical application.

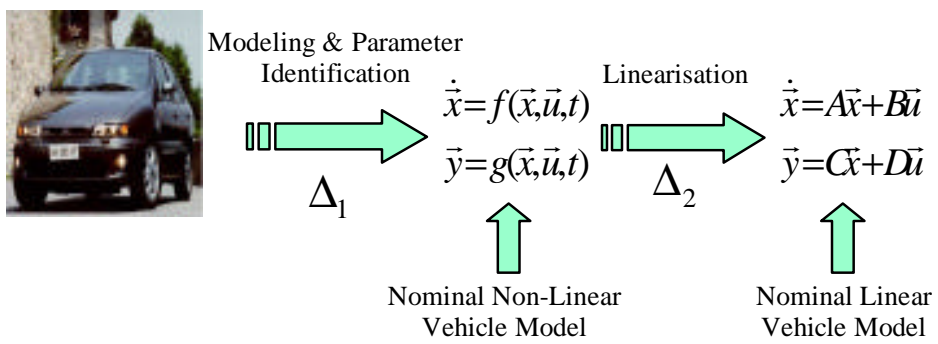

Figure 4 : Modeling error representation

The modeling error $\Delta_{1}$ represents errors in the derivation of the nominal non-linear vehicle model, and the modeling error $\Delta_{2}$ represents errors caused by the linearisation process. Both of these modeling errors may cause the deterioration of Kalman filter performance.

In the Kalman filter design, model uncertainties are included in the design process by supposing the model uncertainties are part of process and measurement noises. It means that the model uncertainties are lumped in the process and measurement noises such that the information about the model uncertainties is lumped in the $Q, R$ and $S$ matrices. To satisfy these requirements, in this study, the process noise $\vec{w}(t)$ and the measurement noise $\vec{v}(t)$ are formulated as the error between source model and linear model. Given random steer angle as described in equation (18), the $\vec{w}(t)$ and $\vec{v}(t)$ data can be generated using the following equation:

$$
\begin{aligned}
& \vec{w}(t)=f(\vec{x}(t), \vec{u}(t), t)-(A \vec{x}(t)+B \vec{u}(t)) \\
& \vec{v}(t)=g(\vec{x}(t), \vec{u}(t), t)-(C \vec{x}(t)+D \vec{u}(t))
\end{aligned}
$$

The $\mathrm{Q}, \mathrm{R}$ and $\mathrm{S}$ matrices are then constructed from $\vec{w}(t)$ and $\vec{v}(t)$ data above using covariance estimator. For any $\mathrm{N}$ data of random variables $\mathrm{x}$ and $\mathrm{y}$, it can be estimated the covariance of $x$ and $y$ using the following covariance estimator:

$$
\hat{c}=(N-1)^{-1}\left(\sum_{k=1}^{N} x_{k} y_{k}-N \hat{m}_{x} \hat{m}_{y}\right)
$$

where

$$
\hat{m}_{x}=N^{-1} \sum_{k=1}^{N} x_{k} \quad \hat{m}_{y}=N^{-1} \sum_{k=1}^{N} y_{k}
$$

Kalman filter can therefore compensate for the magnitude of errors and cross correlation in these magnitudes (between state derivatives and sensors). The problem is that modeling errors are actually time correlated and the method does not take this into account. One key objective of this paper is to understand whether this shortcoming can be reduced to give a better observer if modeling errors are better accounted. Another potential problem is that the Kalman filter accounts for errors as if they were broad bandwidth white noise, whereas in fact the resultant $\vec{w}(t)$ and $\vec{v}(t)$ data bandlimited. It causes misinterpretation of noise behavior such that Kalman filter cannot compensate properly the model uncertainties.

\section{ROBUST FILTER DESIGN}

The alternative, robust filter is a deterministic filter that again based on the linear vehicle model but takes better account of modeling errors through a bounded expectation of error in the system and output matrices. This filter also includes uncorrelated error expectations $\mathrm{w}$ and $\mathrm{v}$, so is in effect an extended Kalman filter. Consider the linear time varying vehicle model that is formulated as follow:

$$
\begin{aligned}
& \dot{x}(t)=(A+\Delta A(t)) x(t)+B u(t)+w(t) \\
& y(t)=(C+\Delta C(t)) x(t)+D u(t)+v(t)
\end{aligned}
$$

where $\quad A \in \mathfrak{R}^{n x n}, \quad B \in \mathfrak{R}^{n x m}, \quad C \in \mathfrak{R}^{p x n}, \quad$ and $D \in \Re^{p x m}, \Delta A(t)$ and $\Delta C(t)$ are the time varying perturbations around the nominal vehicle model which are assumed to be bounded and structured as follows:

$$
\left[\begin{array}{l}
\Delta A(t) \\
\Delta C(t)
\end{array}\right]=\left[\begin{array}{l}
L_{1} \\
L_{2}
\end{array}\right] \Delta(t) N_{1}
$$


where $L_{1} \in \mathfrak{R}^{n x m}, L_{2} \in \mathfrak{R}^{p x m}, N_{1} \in \mathfrak{R}^{q x n}$ are constant matrices and $\Delta(\cdot)$ is assumed to be a piecewise continuous matrix function belonging to the set:

$$
\Omega=\left\{\Delta(\cdot): \Delta(t) \Delta^{\prime}(t)<I_{m}, \forall t\right\}
$$

The process and measurement noises $\vec{w}(t)$ and $\vec{v}(t)$ are assumed to be independent zero mean white noises with unknown time-varying intensities $W(t)$ and $V(t)$ belonging to the uncertainty sets:

$$
\begin{aligned}
& \varpi=\{W(\cdot): W(t) \leq \bar{W}, \forall t, \bar{W} \geq 0\} \\
& \nu=\{V(\cdot): V(t) \leq \bar{V}, \forall t, \bar{V} \geq 0\}
\end{aligned}
$$

The objective of the robust filtering problem is to minimise the upper bound of the filtering error variance as follow:

$$
E\left\{\left[\vec{x}(t)-\vec{x}_{e}(t)\right]\left[\vec{x}(t)-\vec{x}_{e}(t)\right]^{\prime}\right\}=\Pi(t)
$$

Then a robust filter can be formulated with the following structure:

$$
\begin{aligned}
& \dot{x}_{e}(t)=\left(A+\frac{1}{\beta} \Pi N_{1} N_{1}^{\prime}\right) x_{e}(t)+B u(t)+K_{r}\left(y(t)-C x_{e}(t)-D u(t)\right) \\
& K_{r}=\left(\bar{\Pi} C^{\prime}+\beta L_{1} L_{2}^{\prime}\right)\left(\bar{V}+\beta L_{2} L_{2}^{\prime}\right)^{-1}
\end{aligned}
$$

where $K_{r}$ is the robust filter gain, $\beta$ is an arbitrary positive constant and $\Pi$ is the steady state solution of the following algebraic Riccati equation:

$$
\begin{aligned}
& A \bar{\Pi}+\bar{\Pi} A^{\prime}+\frac{1}{\beta} \bar{\Pi} N_{1}^{\prime} N_{1} \bar{\Pi}-\left(\bar{\Pi} C^{\prime}+\beta L_{1} L_{2}^{\prime}\right)\left(\bar{V}+\beta L_{2} L_{2}^{\prime}\right)^{-1}\left(\bar{\Pi} C^{\prime}\right. \\
& \left.+\beta L_{1} L_{2}^{\prime}\right)^{\prime}+\beta L_{1} L_{1}^{\prime}+\bar{W}=0
\end{aligned}
$$

It can be seen from equation (29) that this robust filter has a different structure from common observers, as it has an additional component in the A matrix, $\frac{1}{\beta} \Pi N_{1}^{\prime} N_{1}$. This allows inclusion of vehicle model uncertainties in the filtering computation.

The robust filter provides more tools/parameters to capture the information of model uncertainties than the Kalman filter. With the addition of structured model uncertainty in its design process, it uses more information about the uncertain dynamics around the nominal linear model than the Kalman filter.

In this study, the structured model uncertainties for the vehicle are obtained from simulation using the source model. The source model generates data for vehicle states, state derivatives, input variables, sensor measurements and tyre forces under the same operating conditions that are used in the Kalman filter design in generating $Q, R$ and $S$ matrices. All of these data are then used to generate time varying $A$ and $C$ matrices.

For each point in time, the linear model can be estimated at that point by giving a small change in the states and then calculating the elements of the $\mathrm{A}$ and $\mathrm{C}$ matrices using a numerical Jacobian method as follows:

$$
\begin{aligned}
& A_{i j}(t)=\frac{\Delta f_{i}(t)}{\Delta x_{j}} \quad i=1, \ldots, n \quad j=1, \ldots, n \\
& C_{i j}(t)=\frac{\Delta g_{i}(t)}{\Delta x_{j}} \quad i=1, \ldots, p \quad j=1, \ldots, n
\end{aligned}
$$

where $\mathrm{n}$ is the number of state variables, $\mathrm{p}$ is the number of sensor measurement variables and $\Delta x$ is chosen as some suitably small perturbation-in this study is $10^{-8}$. The elements of $\Delta A(t)$ and $\Delta C(t)$ data then can be obtained by subtracting all of the elements of $A(t)$ and $C(t)$ with its nominal value (as given in equation (15)) at each point in time.

There is no standard procedure to decompose $\Delta A(t)$ and $\Delta C(t)$ to get $L_{1}, L_{2}$ and $N_{1}$. In this study, the decomposition is done in such a way that it takes into account the strong correlation between the $\mathrm{A}$ and $\mathrm{C}$ matrices which exists due to the nature of sensor dynamics as a linear combination of state dynamics. The time varying $\Delta(t)$ matrix is set as a diagonal matrix whose diagonal elements are the normalised form of each $\Delta A(t)$ element as follows:

$$
\begin{aligned}
& \Delta(t)=\operatorname{diag}\left[n a_{11}(t) . . n a_{1 n}(t), n a_{21}(t) . . n a_{2 n}(t), \ldots, n a_{n 1}(t) . . n a_{n n}(t)\right]^{T} \\
& n a_{i j}(t)=\frac{a_{i j}(t)}{\bar{a}_{i j}} ; \bar{a}_{i j}=\max \left(a b s\left(a_{i j}(t)\right)\right) ; i, j=1,2, \ldots, n
\end{aligned}
$$

The $N_{1}$ matrix is set as the scaling factor that normalise $\Delta A(t)$ as follows:

$$
N_{1}=\left[\begin{array}{ccccc}
\bar{a}_{11} & \ldots & 0 & \ldots \ldots . \\
0 & \bar{a}_{12} & \ldots & 0 & \ldots . \\
\ldots \ldots \ldots . & \ldots . & . . \\
\ldots . & 0 & \ldots . & \bar{a}_{1 n} \\
& & & \\
\bar{a}_{n 1} & \ldots .0 & \ldots . . & . \\
\ldots \ldots \ldots . & \ldots \ldots . . \\
\ldots . & 0 & \ldots . . & \bar{a}_{n n}
\end{array}\right]_{n^{2} x n}
$$


$L_{1}$ is then set as coefficient matrix such that equation (28) is satisfied:

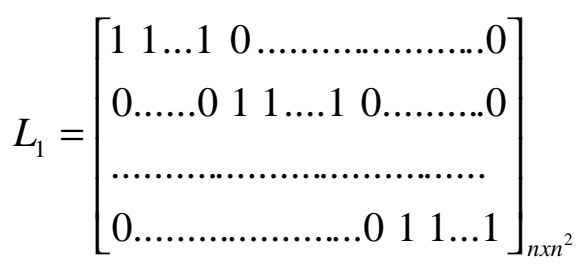

As the sensor and model errors are directly correlated, the elements of $\Delta C(t)$ can all be described as linear combinations of the defined $\Delta(t) . L_{2}$ is thus found by linear regression of the elements of $\Delta C(t)$ against $\Gamma(t)$, formulated as follows:

$$
\begin{gathered}
\Gamma(t)=\Delta(t) N_{1} \\
L_{2}=\left(\left(\Gamma(t) \Gamma(t)^{T}\right)^{-1} \Gamma(t) \Delta C(t)^{T}\right)^{T}
\end{gathered}
$$

where $L_{2} \in R^{p x n^{2}}$.

\section{SIMULATION RESULT}

The design simulation for both the Kalman filter and the robust filter is done under the same operating condition. The vehicle is operated under constant forward speed of $20 \mathrm{~m} / \mathrm{s}$. The source model is excited using a random steer input to provide data of $\vec{w}(t)$ and $\vec{v}(t)$. The test is designed to be achievable in a real vehicle, so the random steer is generated using white noise which is bandlimited within a physically realisable range to $5 \mathrm{~Hz}$ as given in equation (18). Figure 5 shows the steer test input, which is limited to a maximum $3^{\circ}$ steer angle with rms value of 1.7311 degree. The simulation is done for 100 seconds.

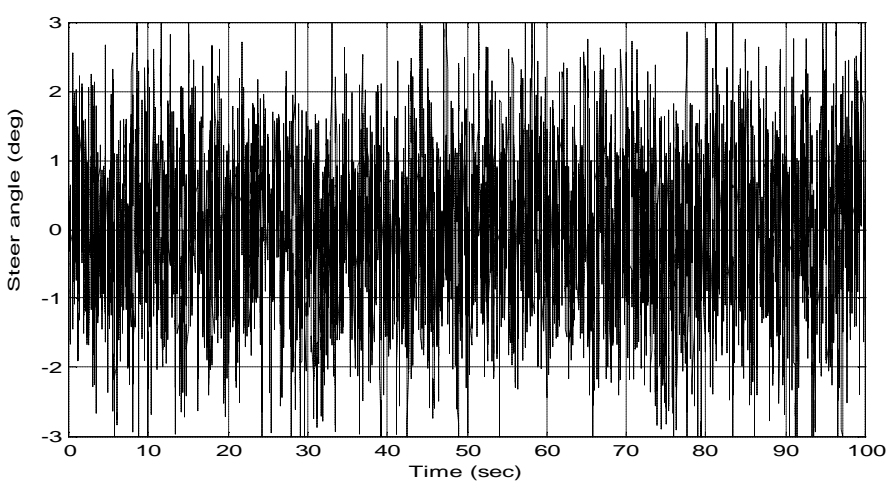

Figure 5 : Random steer angle

The amplification $\rho$ for measurement noise is set to 0.2 to simulate that the rms noise is $20 \%$ of a nominal lateral acceleration of $2 \mathrm{~m} / \mathrm{s}^{2}$. The designs are then conducted and described using equation (22) to (26) for the Kalman filter and equation (28) to (33) for the robust filter. It is important to note that in this simulation, the information about model uncertainties in the Kalman filter are all included in $\mathrm{Q}, \mathrm{R}$ and $\mathrm{S}$ matrices, whereas in the robust filter these are included in $L_{1}, L_{2}, N_{1}$ and $\mathrm{V}$ matrices. To get a comparable result, the $\mathrm{W}$ matrix in the robust filter is set to zero and the $\mathrm{V}$ matrix only contains the mean square value of external measurement noises simulated in equation (13). The parameter $\beta$ for the robust filter is set to 0.76 as this gives the optimum performance index. Both filters are then tested using piecewise continuous steer angle input for 25 seconds described in Figure 6.

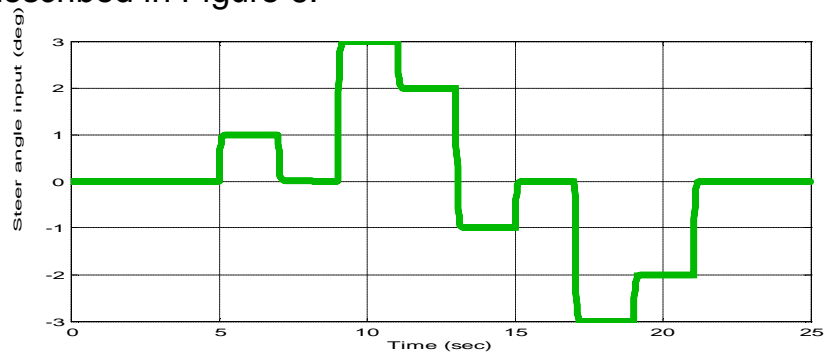

Figure 6 : Piecewise continuous steer angle input

Figure 7 shows the comparison of estimated state trajectories for both the Kalman and robust filters against source model states.
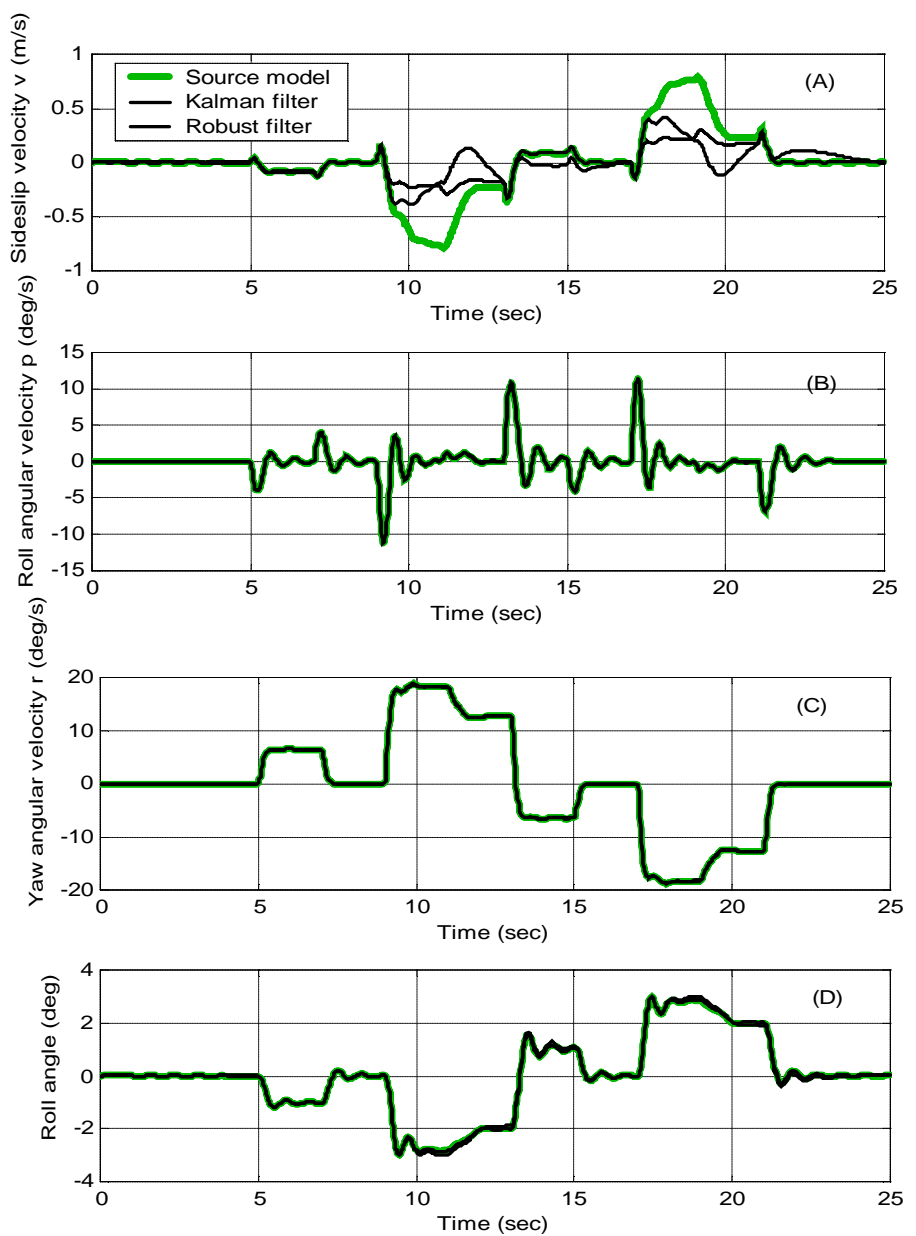

Figure 7 : Performance comparison between Kalman filter \& Robust filter 
The rms value of estimation errors as the percentage of true state rms value is shown in Table 2.

\begin{tabular}{|c|c|c|c|c|}
\hline Filter & $\mathrm{v}$ & $\mathrm{p}$ & $\mathrm{r}$ & $\theta$ \\
\hline Kalman & 70.4577 & 1.2081 & 0.0255 & 4.6487 \\
\hline Robust & 63.1782 & 0.0922 & 0.2703 & 0.8366 \\
\hline
\end{tabular}

\section{Table 2 : RMS error as a percentage of signal}

Table 2 shows that the performance of the robust filter is generally better than that of the Kalman filter. The robust filter has a better estimation result in almost all states except in yaw rate.

Figure 7 shows that both filters estimate roll rate, yaw rate and roll angle extremely well. Analytically, this phenomena is reasonable because the non-linearity of the vehicle model in $p, r$ and $\theta$ states is relatively insensitive to the tyre non-linearity, which in the vehicle model has the biggest non-linearity effect. From equation (3), it can be shown that the roll dynamics are strongly affected by spring-damper forces of the suspension, which are linear in the model. From equation (4), it can be shown that the yaw dynamics are strongly affected by lateral tyre forces. However, since the overall yaw moment is caused by the subtraction between lateral tyre force moment at front wheels and rear wheels, the overall non-linearity affected by lateral tyre forces is reduced. Yaw and roll modes are also well accommodated within the sensor feedback, so nonlinearity errors are rapidly diminished.

The biggest non-linearity effect occurs in sideslip velocity dynamics. Equation (2) shows that sideslip is caused by the addition of all four lateral tyre forces, so this is strongly influenced by tyre non-linearity. Figure 8 shows the lateral tyre force dynamics at the left front wheel and describes how the non-linearity affects the sideslip velocity. As can be shown in Figure 7 plot $A$, both filters can do a good estimation for small steer angle input, but this gets worse when the steer angle is larger. For $5 \leq t \leq 7 \sec \left(\delta=1^{0}\right)$, both filters estimate sideslip very well. This is because in this stage, the sideslip angle exists within the linear region of the lateral tyre forces characteristic. Figure 8 shows that, at this stage, the 'true' lateral tyre force from the source model coincides with the expected lateral tyre force from the linear model. For $7 \leq t \leq 9$ sec $\left(\delta=3^{0}\right)$ and $17 \leq t \leq 19 \sec \left(\delta=-3^{0}\right)$, both filters make a bad estimation, as can be shown by relatively large steady state estimation errors. This estimation error occurs because the sideslip angle has entered the non-linear region of tyre force characteristic. Figure 8 shows that at this stage, the lateral tyre force from linear calculation is bigger than the source tyre force ; the source tyre force has reached a saturated region such that the effective cornering stiffness is less than the linear cornering stiffness, and this is why the source tyre force is smaller than the linear tyre force. Both observers assume that the 'actual' tyre forces are the tyre forces calculated by linear computation. Because lateral tyre forces act in resistance to sideslip velocity, this makes the observers interpret the resistance forces as larger than the actual resistance forces, and this is why the estimated sideslip velocity is less than the source sideslip velocity.

Note also the difference in nature of sideslip errors between the robust and Kalman filters. The robust filter performs quantitatively better than the Kalman filter, because the model is assumed to be more robust, and hence more reliable. Although its estimate is incorrect, the robust filter "believes" its model information during the maximum extreme $\left(3^{0}\right)$ steer condition, and this results in a more stable estimate when the steer angle is relaxed to $2^{0}$. Conversely, the Kalman filter uses a higher feedback of sensor information which causes low frequency drifting - eg between 9 and 13 seconds in Figure 7A. This is because the sideslip velocity is being estimated by integrating acceleration more than by the (unreliable) model.
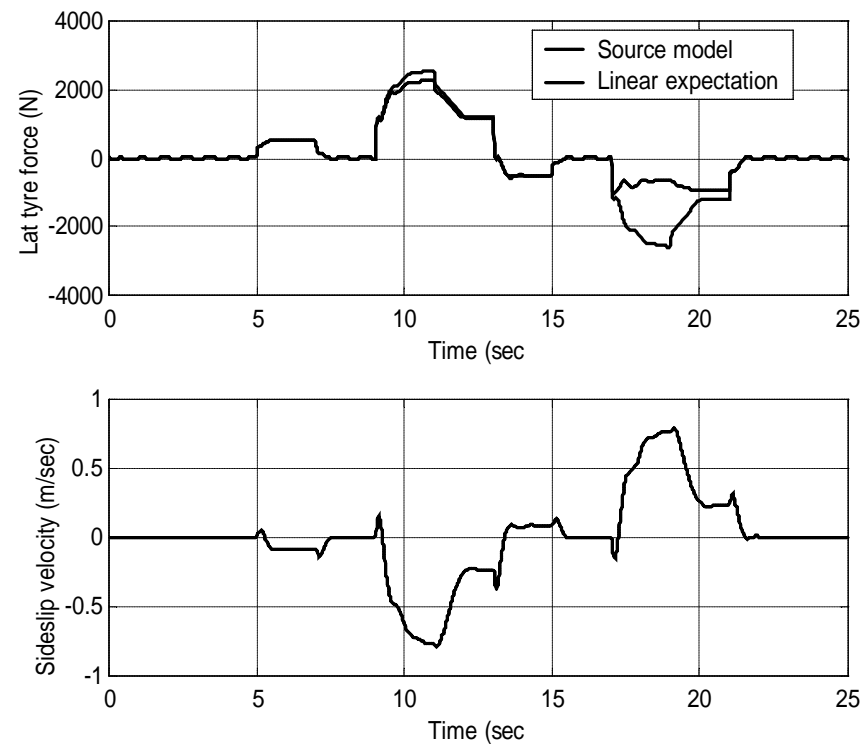

Figure 8 : Lateral tyre force dynamics

To complete the assessment, the performance of both filters is also examined under two different random steer tests $\left(\delta_{1}\right.$ and $\left.\delta_{2}\right)$ using different seed to obtain different $\mathrm{Q}, \mathrm{R}$ and $\mathrm{S}$ covariance matrices for Kalman filter and different $L_{1}, L_{2}$ and $N_{1}$ matrices for robust filter. The estimation result for both filters is summarised in Table 3, and here we also consider the effect of choosing a reduced sensor set. For set $A$, $y_{A}=\left[s_{1}, s_{2}, s_{3}, s_{4}\right]^{T}$ and for set B, $y_{B}=\left[s_{1}, s_{2}, s_{4}\right]^{T}$. 


\begin{tabular}{|c|c|c|c|c|c|}
\cline { 2 - 6 } \multicolumn{1}{c|}{} & \multicolumn{2}{c|}{$\delta_{1}$} & \multicolumn{2}{c|}{$\delta_{2}$} \\
\cline { 2 - 6 } \multicolumn{1}{c|}{} & State & Kalman & Robust & Kalman & Robust \\
\hline \multirow{3}{*}{ Set A } & $\mathrm{v}$ & 64.2992 & 62.6253 & 64.2803 & 62.6285 \\
\cline { 2 - 6 } & $\mathrm{p}$ & 0.353 & 0.0013 & 0.3633 & 0.0013 \\
\cline { 2 - 6 } & $\mathrm{r}$ & 6.7535 & $1.25 \mathrm{E}-04$ & 6.7557 & $1.25 \mathrm{E}-04$ \\
\cline { 2 - 6 } & $\theta$ & 0.2367 & 0.1377 & 0.2381 & 0.1377 \\
\hline \multirow{3}{*}{ Set B } & $\mathrm{V}$ & 64.4514 & 62.9474 & 64.4448 & 62.9473 \\
\cline { 2 - 6 } & $\mathrm{p}$ & 22.0299 & 18.8093 & 21.5287 & 18.8092 \\
\cline { 2 - 6 } & $\mathrm{r}$ & 3.8519 & 2.1277 & 3.8186 & 2.1277 \\
\cline { 2 - 6 } & $\theta$ & 2.4892 & 6.1038 & 2.4669 & 6.1038 \\
\hline
\end{tabular}

Table 3 : RMS error as a percentage of signal

The performance of the robust filter for both $\delta_{1}$ and $\delta_{2}$ and both sensor set $A$ and $B$ is again better than that of the Kalman filter. Both filters show performance deterioration for sensor set $B$ rather than sensor set $A$, this is because the information obtained is sensor set $B$ is less than that of in sensor set $A$. Note that estimation of both yaw and roll modes is slightly worse because these modes are now being sensed in combination by $S_{2}$ and $S_{4}$ whereas yaw is independent from roll in sensor set $A$ (sensed by $S_{s}-S_{3}$ and $S_{3}-S_{4}$ respectively). The performance degradation is small enough to make the reduced sensor set attractive however. The accuracy of sideslip estimation is not affected (or improved) by the choice of set B.

\section{PRACTICAL VIABILITY}

There are a number of issues which would need to be resolved before any practically viable observer could be developed. The most significant issues are related to the construction of noise covariance matrices in Kalman filter design and structured model uncertainty matrices in robust filter design, since these tools are the main factors which influence filter performance. The estimation for both noise covariance matrices and structured model uncertainty matrices idealised in this study. Unfortunately, practical estimation is very difficult without elaborate instrumentation of a test vehicle. The best solution will probably be achieved by combining the test data from on-line and real time test and the data from off-line simulation to get nominal noise covariance matrices and structured uncertainty matrices. These nominal matrices could then be tuned manually or automatically using a suitable adaptation algorithm.

The other issue is related to the non-linearity of tyre characteristic which gives significant effect to the filter performance deterioration. This problem is likely can be solved by utilised cornering stiffness adaptation in the observer such that the expected tyre force by linear computation adapts the actual tyre force. Alternatively, the use of non-linear, extended Kalman filter should be feasible. These might be very accurate in the robust form motivated here.
Finally, since this study still deals with continuous filters, it is important to examine the performance of both filters in their respective discrete time forms. The issue of time sampling has to be taken into account since this will also affect the filter performance.

\section{CONCLUSION}

Linear filter methods have been proposed to estimate vehicle handling dynamics states. Within a simulation environment and under similar operating conditions, robust filter shows better performance than Kalman filter. The time-correlated nature of vehicle model uncertainties is better compensated by the robust filter rather than the Kalman filter because the former includes time varying parameter dynamics directly in constructing structured model uncertainties. However, the Kalman filter has a better ability to interpret broadband noises, since it assumes that the noise statistic is well known, whereas the robust filter assumes that this statistic is unknown. This fact brings the conclusion that best observer may be designed by combining the ability of the robust filter to interpret the model uncertainties and the ability of Kalman filter to interpret noise characteristic, in a robust Kalman filter.

\section{ACKNOWLEDGMENTS}

This paper is a part of $\mathrm{PhD}$ research conducted by the principal author in the Department of Aeronautical and Automotive Engineering, Loughborough University, UK.

\section{REFERENCES}

1. T. J. Gordon, "An Integrated Strategy for the Control of a Full Vehicle Suspension System," Vehicle System Dynamics, vol. 25, pp. 229-242, 1996.

2. L. Austin and D. Morrey, "Recent Advances in Antilock Braking Systems and Traction Control Systems," Proceedings of the Institution of Mechanical Engineering, vol. 214, pp. 625-638, 2000.

3. S. M. El-Demerdash, A. M. Sell, and D. A. Cron, "Vehicle Body Attitude Control Using an Electronically Controlled Active Suspension," Society of Automotive Engineers, pp. 1232-1242, 1999.

4. H. Kawakami, H. Sato, M. Tabata, H. Inoue, and H. Itimaru, "Development of Integrated System Between Active Control Suspension, Active 4WS, TRC and ABS," SAE Special Publications, vol. 917, pp. 41-48, 1992.

5. Y. Fukada, "Estimation of Vehicle Slip-angle with Combination Method of Model Observer and Direct Integration," Proceedings of the International 
Symposium on Advanced Vehicle Control 1998, pp. 201-206, 1998.

6. U. Kienecke and A. Daib, "Observation of Lateral Vehicle Dynamics," Control Engineering Practice, vol. 5, pp. 1145-1150, 1997.

7. P. J. T. Venhovens and K. Naab, "Vehicle Dynamics Estimation Using Kalman Filters," Vehicle System Dynamics, vol. 32, pp. 171-184, 1999.

8. M. C. Best, T. J. Gordon, and P. J. Dixon, "An Extended Adaptive Kalman Filter for Real-time State Estimation of Vehicle Handling Dynamics," Vehicle System Dynamics, 2000.

9. M. C. Best and T. J. Gordon, "Real Time State Estimation of Vehicle Handling Dynamics Using an Adaptive Kalman Filter," Proceedings of the International Symposium on Advanced Vehicle Control 1998, pp. 183-188, 1998.

10. W. F. Milliken and D. L. Milliken, Race Car Vehicle Dynamics: SAE International, 1995.

11. B. D. O. Anderson and J. B. Moore, Optimal Control : Linear Quadratic Methods: Prentice Hall Inc, 1990.

12. P. Bolzern, P. G. Colaneri, and D. Nicolao, "Optimal Robust Filtering with Time-varying Parameter Uncertainty," International Journal of Control, vol. 63, pp. 557-576, 1996.

13. C. W. Helstrom, Probability and Stochastic Process for Engineers, Second Edition ed: Macmillan Publishing Company, 1991.

\section{CONTACT}

Medy Satria, PhD student in Department of Aeronautical and Automotive Engineering, Loughborough University, Ashby Road, Loughborough, Leicestershire, LE11 3TU, United Kingdom. E-mail :m.satria@lboro.ac.uk 